\title{
ANALYTICAL TREATMENT FOR NONLINEAR OSCILLATION EQUATIONS AND VIBRATORY SYSTEM OF WAVES
}

\author{
M. NAJAFI, M. MOGHIMI AND H. MASSAH
}

\begin{abstract}
Analytical approximate solutions of Duffing and Van der Pol equations as well as the system of coupled Euler-Bernoulli beams and wave equations are under consideration. To this end, the Adomian Decomposition Method (ADM) and variational iteration method (VIM) have been employed to obtain analytical solutions to these differential equations. The results are compared with accurate numerical computations, which show that ADM is a high performance and accurate method to use for the analytical solution of nonlinear physical problems.
\end{abstract}

\section{Introduction}

Achievement of the exact solution for nonlinear equations is an ambitious and perfect goal for engineers, scientists, and mathematicians. However, computational finite discrete approaches such as Finite Volume Method, Finite Difference Method, and Finite Element Methods have been widely used to solve any physical problem in the past decades. As an example, computational methodologies have been the only way to solve the Navier-Stokes (N-S) equation (White, 1991) for predicting flow behavior in most flows.

Any type of approximate solution would be very valuable for mathematicians and engineers. To this end, the Adomian Decomposition Method (ADM) by Adomian (1988) is one the techniques which was introduced to solve nonlinear ordinary or partial differential equations. An advantage of this method is that it can provide an approximate solution to a rather wide class of nonlinear (and stochastic) equations without linearization, perturbation, closure approximation, or discretization methods. Unlike the common methods, i.e., weak nonlinearity and small perturbation which are changing the physics of the problem due to simplification, ADM gives the approximate analytical solution of the problem without any simplification. Thus, the results are more realistic. Recently, several researchers have used ADM to solve a wide range of physical phenomena (Adomian (1994), He (1997, 1998, 1999) and Pamuk (2005)) in various engineering and science fields.

In this work, the effort has been made to find the analytical solutions, via ADM and VIM, of oscillating systems in engineering and science, respectively, which are represented by $m \ddot{u}+$ $f(u, \dot{u}, c, k)=0$. By employing an especial approximation for restoring force, $f(u)$, Duffing's differential equation produces results, which models a large number of dynamic systems. The

Received April 16, 2007.

Key words and phrases. Analytic solution, Adomian decomposition method, nonlinear Oscillation, Duffing equation, Van der Pol equation, Runge-Kutta, wave equation. 
Duffing double-well oscillator was first developed to model forced vibrations of industrial machinery. Even though Van der Pol's equation was originally developed to describe the dynamics of a triode electronic oscillation, it demonstrates many of the basic properties of a nonlinear system representing a mechanical self-exited mechanism. For nonlinear models, the methods have shown reliable results in analytical approximation that converges very rapidly (Abbaoui, K. and Cherruault, Y. (1999)). Finally, devotion goes to searching for an approximate solution of a system of coupled Euler-Bernoulli beams and wave equations analytically via ADM.

This paper is organized as follows: in Section 2, the basics and application of ADM as well as VIM will be discussed. Approximate solutions of Duffing and Van der Pol equations will be found analytically via ADM and VIM in Sections 3 and 4, respectively. In Section 5, numerical computations are pursued via the Runge-Kutta method in order to compare the results. Sections 6 and 7 devote to the applications of ADM for partial differential equations, i.e., the system of Euler-Bernoulli beam equations and wave equations in parallel followed by the conclusion in Section 8.

\section{Basics and application of ADM and VIM}

The principal algorithm of ADM when applied to a general nonlinear equation is in the following form by Adomian (1994):

$$
L u+R u+N u=g .
$$

The linear terms are decomposed into $L+R$, while the nonlinear terms are represented by $N u$. Note, $L$ is taken as the highest order derivative to avoid difficult integration involving complicated Green's functions, and $R$ is the remainder of the linear operator. In addition, $L^{-1}$ is a definite integration from 0 to $t$, i.e.,

$$
L^{-1}[\cdot]=\int_{0}^{t} \int_{0}^{t}[\cdot] d t d t .
$$

Obviously, if $L$ is a second-order operator, $L^{-1}$ is a two-fold indefinite integral as:

$$
L^{-1} L u=u(x, t)-u(x, 0)-\frac{\partial u(x, 0)}{\partial t} t .
$$

Operating on both sides of (1) with $L^{-1}$ yields:

$$
L^{-1} L u=L^{-1} g-L^{-1} R u-L^{-1} N u,
$$

and finally

$$
u(x, t)=u(x, 0)+u_{t}(x, 0) t+L^{-1} g-L^{-1} R u-L^{-1} N u .
$$

The decomposition method represents the solution of (4) as the following series is:

$$
u(x, t)=\sum_{n=0}^{\infty} u_{n}(x, t)
$$


The non-linear operator $N u$ is decomposed as

$$
N u=\sum_{n=0}^{\infty} P_{n} .
$$

Substituting (6) and (7) into (5), one gets

$$
\sum_{n=0}^{\infty} u_{n}(x, t)=u_{0}-L^{-1} R \sum_{n=0}^{\infty} u_{n}-L^{-1} \sum_{n=0}^{\infty} P_{n},
$$

where

$$
u_{0}=u(x, 0)+u_{t}(x, 0) t+L^{-1} g .
$$

Consequently, Equation (8) can be calculated as:

$$
\begin{aligned}
& u_{1}=-L^{-1} R u_{0}-L^{-1} P_{0}, \\
& u_{2}=-L^{-1} R u_{1}-L^{-1} P_{1}, \\
& \vdots \\
& u_{n}=-L^{-1} R u_{n}-L^{-1} P_{n}, \quad n \geq 0,
\end{aligned}
$$

where $P_{n}$ are the Adomian's polynomials of $u_{0}, u_{1}, \ldots, u_{n}$ and are obtained from the following formula by Adomian (1988):

$$
P_{n}=\frac{1}{n !} \frac{d^{n}}{d \lambda^{n}}\left[F\left(\sum_{i=0}^{\infty} \lambda^{i} u_{i}\right)\right]_{\lambda=0}, \quad n=0,1,2, \ldots
$$

Equation (11) gives:

$$
\begin{aligned}
& P_{0}=f\left(u_{0}\right), \\
& P_{1}=u_{1} \frac{d}{d u_{0}} f\left(u_{0}\right), \\
& P_{2}=u_{2} \frac{d}{d u_{0}} f\left(u_{0}\right)+\frac{u_{1}^{2}}{2 !} \frac{d^{2}}{d u_{0}^{2}} f\left(u_{0}\right), \\
& P_{3}=u_{3} \frac{d}{d u_{0}} f\left(u_{0}\right)+u_{1} u_{2} \frac{d^{2}}{d u_{0}^{2}} f\left(u_{0}\right)+\frac{u_{1}^{3}}{3 !} \frac{d^{3}}{d u_{0}^{3}} f\left(u_{0}\right),
\end{aligned}
$$

The accuracy level of the approximation of $u(x, t)$ in (6) can be dramatically enhanced by computing coefficients (as many as we would like). Here, $u_{0}$ can be obtained using initial conditions; and, consequently, all of $u_{n}$ are calculable. Since the series conveges very rapidly, the $k$-term approximation can be used as a practical solution:

$$
\varphi_{k}=\sum_{n=0}^{k-1} u_{n}, \text { where } \lim _{k \rightarrow \infty} \varphi_{k}=u .
$$

Convergence has been rigorously established (Abbaoui, K. and Cherruault, Y. (1999)). 


\subsection{Variational iteration method}

Having considered (1) and to illustrate the basic concepts of the variational iteration method, one can, according to He's variational iteration method (1999), construct a correction functional as follows:

$$
u_{u+1}(t)=u_{n}(t)+\int_{0}^{t} \lambda\left[L u_{n}(\tau)+N \overline{u_{n}(\tau)}-g(\tau)\right] d \tau
$$

where $\lambda$ is a general Lagrangian multiplier, which can be identified optimally via the variational theory, the subscript $n$ denotes the $n$th order approximation, $\overline{u_{n}}$ is considered as a restricted variation, i.e., $\overline{u_{n}}=0$. Equation (14) is called a correction functional. The variational iteration method proposed by He, J. H., (1999), has been shown to solve effectively, easily, and accurately a large class of nonlinear problems with approximations converging repidly to accurate solutions. For linear problems, its exact solution can be obtained by only one iteration step due to the fact that the Lagrange multiplier can be exactly identified.

\section{Solution to Duffing equation via ADM and VIM}

Now in this section, we are in the position to apply these methods, ADM and VIM, to our nonlinear differential equations as well as to partial differential equation in Section 6 .

The following problem is motivated by an analogous problem in ordinary differential equations and widely used in many perturbation techniques:

$$
\ddot{u}+u+\varepsilon u^{3}=0
$$

with initial onditions,

$$
u(0)=1, \quad \dot{u}(0)=5 .
$$

Rewriting (15) in the following operator form:

$$
L_{t}(u)+u+\varepsilon N(u)=0 .
$$

The highest order linear derivative operator is $L_{t}$, where $L_{t}$ is a second order differential operator with respect to $t$. The nonlinear operator is represented by $N$. The integral operator, $L_{t}^{-1}$, is a twofold integration with respect to $t$. Applying the $L_{t}^{-1}$ to (17) and using initial conditions in (16) yields

$$
u(t)=1+5 t-L_{t}^{-1}(u)-\varepsilon L_{t}^{-1} N(u) .
$$

The solution, $u(t)$, can be decomposed as

$$
u(t)=\sum_{n=0}^{\infty} u_{n}(t)
$$

Substituting (19) into (18) gives

$$
\sum_{n=0}^{\infty} u_{n}(t)=1+5 t-L_{t}^{-1}\left(\sum_{n=0}^{\infty} u_{n}(t)\right)-\varepsilon L_{t}^{-1} N\left(\sum_{n=0}^{\infty} u_{n}(t)\right) .
$$


Equation (20) is transformed to a set of recursive relations given by

$$
\left\{\begin{array}{l}
u_{0}(t)=1+5 t \\
u_{n+1}(t)=-L_{t}^{-1}\left(u_{n}\right)-\varepsilon L_{t}^{-1} N\left(u_{n}\right), \quad n \geq 0
\end{array}\right.
$$

where $u_{0}$ is obtained using initial conditions and the $u_{n+1}$ terms are calculated using the preceding relations. Hence, the summation of the $u_{n}$ terms is the desired solution, which converges rapidly. In practice, we can calculate the first $k$ terms of the summation in place of the whole. Therefore, the approximate solution is

$$
\varphi_{k}=\sum_{n=0}^{k-1} u_{n}(t) .
$$

The procedure is clear to follow and to calculate. Below, you will find the first two terms of the analytical approximation to (15):

$$
\begin{aligned}
u_{1}(t)= & -\frac{1}{2} t^{2}-\frac{5}{6} t^{3}-\frac{1}{500} \varepsilon(1+5 t)^{5}, \\
u_{2}(t)= & \left(\frac{3}{1000} t^{2}+\frac{7}{200} t^{3}+\frac{21}{80} t^{4}+\frac{21}{16} t^{5}+\frac{35}{8} t^{6}+\frac{75}{8} t^{7}+\frac{375}{32} t^{8}+\frac{625}{96} t^{9}\right) \varepsilon^{2} \\
& +\left(\frac{1}{1000} t^{2}+\frac{1}{120} t^{3}+\frac{1}{6} t^{4}+t^{5}+\frac{55}{24} t^{6}+\frac{275}{168} t^{7}\right) \varepsilon+\frac{1}{24} t^{4}+\frac{1}{24} t^{5}, \\
u_{3}(t)= & \cdots,
\end{aligned}
$$

So, the result can be presented as a series solution in the form of

$$
\varphi=\sum_{n=0}^{\infty} u_{n}(t)=u_{0}(t)+u_{1}(t)+u_{2}(t)+u_{3}(t)+\cdots .
$$

Summation of the first three-term of $u_{n}(t)$, as in (22), gives a partial sum solution in a series form as follows:

$$
\begin{aligned}
\varphi_{3}=\sum_{n=0}^{2} u_{n}(t)= & -\frac{1}{2} t^{2}-\frac{5}{6} t^{3}-\frac{1}{500} \varepsilon(1+5 t)^{5} \\
& +\left(\frac{3}{1000} t^{2}+\frac{7}{200} t^{3}+\frac{21}{80} t^{4}+\frac{21}{16} t^{5}+\frac{35}{8} t^{6}+\frac{75}{8} t^{7}+\frac{375}{32} t^{8}+\frac{625}{96} t^{9}\right) \varepsilon^{2} \\
& +\left(\frac{1}{1000} t^{2}+\frac{1}{120} t^{3}+\frac{1}{6} t^{4}+t^{5}+\frac{55}{24} t^{6}+\frac{275}{168} t^{7}\right) \varepsilon+\frac{1}{24} t^{4}+\frac{1}{24} t^{5},
\end{aligned}
$$

In practice, due to the rapid convergence of the solution, the first few terms will provide the required accuracy.

\subsection{Duffing solution via VIM}

Following the variational iteration method in Section 2, its correction variational functional in $t$-direction can be expressed as follows:

$$
u_{n+1}(t)=u_{n}(t)+\int_{0}^{t} \lambda\left[\frac{d^{2} u_{n}}{d \tau^{2}}+\overline{u_{n}}+\varepsilon \overline{u_{n}^{3}}\right] d \tau
$$


Here $\delta \overline{u_{n}}$ is considered as a restricted variation, i.e., $\delta \overline{u_{n}}=0$. Making the correction functional, Equation (25), stationary, noticing the $\delta \overline{u_{n}}=0$,

$$
\begin{aligned}
& \delta u_{n+1}(t)=\delta u_{n}(t)+\delta \int_{0}^{t} \lambda\left[\frac{d^{2} u_{n}}{d \tau^{2}}+\overline{u_{n}}+\varepsilon \overline{u_{n}^{3}}\right] d \tau, \\
& \delta u_{n+1}(t)=\delta u_{n}(t)+\delta \int_{0}^{t} \lambda\left[\frac{d^{2} u_{n}}{d \tau^{2}}\right] d \tau, \\
& \delta u_{n+1}(t)=\delta u_{n}(t)\left(1-\lambda^{\prime}(\tau)\right)+\delta \frac{d^{2} u_{n}}{d \tau^{2}} \lambda(\tau)+\int_{0}^{t} \delta u_{n}(\tau) \lambda^{\prime n}(\tau) d \tau=0,
\end{aligned}
$$

yields the following stationary conditions:

$$
\begin{gathered}
\delta u_{n}: 1-\lambda^{\prime}(\tau)=0 \\
\delta \frac{d u_{n}}{d \tau}: \lambda(\tau)=0 \\
\delta u_{n}: \lambda^{\prime \prime}(\tau)=0
\end{gathered}
$$

Therefore, the Lagrange multiplier can be identified as

$$
\lambda(\tau)=\tau-t .
$$

As a result, we obtain the following iteration formula in $t$-direction:

$$
u_{n+1}(t)=u_{n}(t)+\int_{0}^{t}(\tau-t)\left\{\frac{d^{2} u_{n}}{d \tau^{2}}+u_{n}+\varepsilon u_{n}^{3}\right\} d \tau .
$$

We start with the initial condition given by (16), one can find for $u_{0}$

$$
u_{0}(t)=1+5 t
$$

Now, using (31), the following results can be obtained via symbolic packages such as Maple:

$$
\begin{aligned}
& u_{0}=1+5 t, \\
& u_{1}=1+5 t-\frac{25}{4} \varepsilon t^{2}-\frac{25}{4} \varepsilon t^{4}-\frac{5}{6} t^{3}-\frac{5}{2} \varepsilon t^{3}-\frac{1}{2} t^{2}-\frac{1}{2} \varepsilon t^{2}, \\
& u_{2}=\cdots,
\end{aligned}
$$

In Section 5, you will find the comparison of these two methods with its corresponding numerical computation for Duffing Equation.

\section{Solution of van der Pol equation by ADM and VIM}


This equation, which arises in the study of circuits containing vacuum tubes and widely used in many perturbation techniques, is describing self-sustaining oscillations in which energy is fed into small oscillations and removed from large oscillations,

$$
\ddot{u}+u-\varepsilon\left(1-u^{2}\right) \dot{u}=0,
$$

with initial conditions,

$$
u(0)=2, \quad \dot{u}=3 .
$$

Rewriting the equation in the following operator form:

$$
L_{t}(u)+u+\varepsilon N(u)=0 .
$$

Following procedures in Section 2, one can find the analytical solution for (32) as

$$
u(t)=2+3 t-L_{t}^{-1}(u)-\varepsilon L_{t}^{-1} N(u) .
$$

According to ADM, $u(t)$ can be decomposed to

$$
u(t)=\sum_{n=0}^{\infty} u_{n}(t)
$$

Introducing (36) into (35) gives

$$
\sum_{n=0}^{\infty} u_{n}(t)=2+3 u-L_{t}^{-1}\left(\sum_{n=0}^{\infty} u_{n}(t)\right)-\varepsilon L_{t}^{-1} N\left(\sum_{n=0}^{\infty} u_{n}(t)\right) .
$$

The following sets of relations are recursive and acquired by applying ADM to (37):

$$
\left\{\begin{array}{l}
u_{0}(t)=2+3 t \\
u_{n+1}(t)=-L_{t}^{-1}\left(u_{n}\right)-\varepsilon L_{t}^{-1} N\left(u_{n}\right), \quad n \geq 0
\end{array}\right.
$$

By employing the initial conditions, $u_{0}$ is calculated and the other terms, $u_{n+1}$, are obtained recursively using the preceding result. The final solution is the summation of $u_{n}$ terms, which converges quickly. Because of its behavior, that is, its fast convergence, it will suffice to calculate only the first $k$ terms as the solution:

$$
\varphi_{k}=\sum_{n=0}^{k-1} u_{n}(t)
$$

Maple $V$ is utilized to calculate $u_{n}$ 's in symbolic fashion as follows:

$$
\begin{aligned}
u_{1}(t)= & -\frac{9}{4} \varepsilon t^{4}-\frac{1}{6}(3+36 \varepsilon) t^{3}-t^{2}-\frac{9}{2} \varepsilon t^{2}, \\
u_{2}(t)= & \frac{81}{28} \varepsilon^{2} t^{7}+\left(\frac{33}{40} \varepsilon+\frac{27}{2} \varepsilon^{2}\right) t^{6}+\left(\frac{1}{40} \varepsilon+\frac{33}{10} \varepsilon+\frac{477}{20} \varepsilon^{2}\right) t^{5}+\left(\frac{15}{4} \varepsilon+\frac{1}{12} \varepsilon+18 \varepsilon^{2}\right) t^{4} \\
& +\left(\varepsilon+\frac{9}{2} \varepsilon^{2}\right) t^{3} \\
u_{3}= & \cdots,
\end{aligned}
$$


Summation of the first three-terms of $u_{n}(t)$, as in (40), gives a partial sum solution in a series form as follows:

$$
\begin{aligned}
\varphi_{3}=\sum_{n=0}^{2} u_{n}(t)= & 2+\frac{81}{28} \varepsilon^{2} t^{7}+\left(\frac{33}{40} \varepsilon+\frac{27}{2} \varepsilon^{2}\right) t^{6}+\left(\frac{1}{40}+\frac{33}{10} \varepsilon+\frac{477}{20} \varepsilon^{2}\right) t^{5} \\
& +\left(\frac{3}{2} \varepsilon+\frac{1}{12}+18 \varepsilon^{2}\right) t^{4}+\left(-5 \varepsilon+\frac{9}{2} \varepsilon^{2}-\frac{1}{2}\right) t^{3}-\left(1+\frac{9}{2} \varepsilon\right) t^{2}+3 t
\end{aligned}
$$

\subsection{Van der Pol solution by VIM}

According to the variational iteration method, its correction variational functional can be expressed as follows:

$$
u_{n+1}(t)=u_{n}(t)+\int_{0}^{t} \lambda\left[\frac{d^{2} u_{n}}{d \tau^{2}}+\overline{u_{n}}-\overline{\varepsilon\left(1-u_{n}^{2}\right) \frac{d u_{n}}{d \tau}}\right] d \tau .
$$

Here $\delta \overline{u_{n}}$ is considered as a restricted variation, i.e., $\delta \overline{u_{n}}=0$. Making the correction functional, Equation (42), stationary, noticing that $\delta \overline{u_{n}}=0$.

$$
\begin{aligned}
& \delta u_{n+1}(t)=\delta u_{n}(t)+\delta \int_{0}^{t} \lambda\left[\frac{d^{2} u_{n}}{d \tau^{2}}+\overline{u_{n}}-\overline{\varepsilon\left(1-u_{n}^{2}\right) \frac{d u_{n}}{d \tau}}\right] d \tau \\
& \delta u_{n+1}(t)=\delta u_{n}(t)+\delta \int_{0}^{t} \lambda\left[\frac{d^{2} u_{n}}{d \tau^{2}}\right] d \tau \\
& \delta u_{n+1}(t)=\delta u_{n}(t)\left(1-\lambda^{\prime}(\tau)\right)+\delta \frac{d^{2} u_{n}}{d \tau^{2}} \lambda(\tau)+\int_{0}^{t} \delta u_{n}(\tau) \lambda^{\prime \prime}(\tau) d \tau=0
\end{aligned}
$$

yields the following stationary conditions:

$$
\begin{gathered}
\delta u_{n}: 1-\lambda^{\prime}(\tau)=0 \\
\delta \frac{d u_{n}}{d \tau}: \lambda(\tau)=0 \\
\delta u_{n}: \lambda^{\prime \prime}(\tau)=0
\end{gathered}
$$

Therefore, the Lagrange multiplier, therefore, can be identified as

$$
\lambda(\tau)=\tau-t
$$

Hence, the following iteration formulae in $t$-direction will be obtained:

$$
u_{n+1}(t)=u_{n}(t)+\int_{0}^{t}(\tau-t)\left\{\frac{d^{2} u_{n}}{d \tau^{2}}+u_{n}-\varepsilon\left(1-u_{n}^{2}\right) \frac{d u_{n}}{d \tau}\right\} d \tau
$$

When we start with the initial condition given by (33), one gets for $u_{0}$

$$
u_{0}=2+3 t
$$


The following results can be obtained by (48):

$$
\begin{aligned}
& u_{0}=2+3 t \\
& u_{1}=2-\frac{9}{4} \varepsilon t^{4}-\left(\frac{1}{2}+6 \varepsilon\right) t^{3}-\left(\frac{9}{2} \varepsilon+1\right) t^{2}+3 t \\
& u_{2}=\cdots
\end{aligned}
$$

\section{Numerical calculations}

$\mathrm{ADM}$ and VIM results for Duffing and Van der Pol equations are presented in Tables 1-5. Tables 1 and 2 contain the ADM and VIM results for various points in time for $\varepsilon=0.1$. As seem in Tables 1 and 3, the 3-term and 4-term ADM solutions for both of the aforementioned equations are in good agreement with the corresponding numerical results. In addition, in Table 2 and 4, the results of two iterations of VIM are presented. It was observed that when $t$ increases the error is increased too but not in ADM results. It is evident that computation of more terms would result in better approximation. In Table 5, the results of the Van der Pol equation using ADM with $\varepsilon=10$ are compared with those VIM of the 2 -iterations solution. It is shown that $\mathrm{ADM}$ is better than VIM.

\section{Application of ADM for PDE}

In the following two sections, we would like to apply ADM method to the system of linear partial differential equations, i.e., Euler-Benoulli beams as well as the system of waves in $\mathfrak{R}^{2}$, respectively. These problems are motivated by an analogous problem in ordinary differential equations for coupled oscillators and has potential application in isolating a vibrating object from the outside disturbances. For example, rubber or rubberlike materials can be used to either absorb or shield a structure from vibration. As an approximation, thses materials can be modeled as distributed springs. For further applications of such a configuration, interested readers are referred to Najafi, M., (1997 and 2001).

Table 1. Comparison of the numerical results with ADM solution for Duffing equation at $\varepsilon=$ 0.1 for different values of $t$.

\begin{tabular}{|c|c|c|c|c|c|}
\hline$t$ & $\begin{array}{c}3 \text { terms of } \\
\text { ADM }\end{array}$ & $\begin{array}{c}4 \text { terms of } \\
\text { ADM }\end{array}$ & $\begin{array}{c}\text { Numerical } \\
\text { (Runge-Kutta) }\end{array}$ & $\begin{array}{c}\text { Numerical solution - } 4 \\
\text { terms ADM }\end{array}$ & $\begin{array}{c}\text { Absolute Error } \\
\text { \%) }\end{array}$ \\
\hline 0 & 0.99980019 & 0.99980019 & 1.00000000 & 0.00374053 & 0.37405285 \\
\hline 0.001 & 1.00479465 & 1.00479465 & 1.00499941 & 0.00376856 & 0.37498171 \\
\hline 0.01 & 1.04969418 & 1.04969418 & 1.04994019 & 0.00399432 & 0.38043308 \\
\hline 0.1 & 1.49265918 & 1.49265918 & 1.49314227 & 0.00234472 & 0.15703271 \\
\hline 0.5 & 3.18063997 & 3.18068685 & 3.17654735 & 0.00413950 & 0.13031443 \\
\hline 1 & 3.71608038 & 3.83005787 & 3.79760816 & 0.03244971 & 0.85447748 \\
\hline
\end{tabular}


Table 2. Comparison of the numerical results with VIM solution for Duffing equation at $\varepsilon=0.1$ for different values of $t$.

\begin{tabular}{|c|c|c|c|c|}
\hline$t$ & $\begin{array}{c}2 \text { iterations of } \\
\text { VIM }\end{array}$ & $\begin{array}{c}\text { Numerical } \\
\text { (Runge-Kutta) }\end{array}$ & $\begin{array}{c}\text { Numerical solution - } \\
\text { iterations of VIM }\end{array}$ & $\begin{array}{c}\text { Absolute Error } \\
(\%)\end{array}$ \\
\hline 0 & 1 & 1.00000000 & 0 & 0 \\
\hline 0.001 & 1.004999 & 1.00499941 & -0.00000004 & 0.00000398 \\
\hline 0.01 & 1.049944 & 1.04994019 & -0.00000372 & 0.00035431 \\
\hline 0.1 & 1.493348 & 1.49314227 & -0.00020565 & 0.01377274 \\
\hline 0.5 & 3.16849 & 3.17654735 & 0.00805777 & 0.25366430 \\
\hline 1 & 3.116667 & 3.79760816 & 0.68094149 & 17.93079919 \\
\hline
\end{tabular}

Table 3. Comparison of the numerical results with ADM solution for Van der Pol equation at $\varepsilon=0.1$ for different values of $t$.

\begin{tabular}{|c|c|c|c|c|c|}
\hline$t$ & 3 terms of ADM & $\begin{array}{c}4 \text { terms of } \\
\text { ADM }\end{array}$ & $\begin{array}{c}\text { Numerical } \\
\text { (Runge-Kutta) }\end{array}$ & $\begin{array}{c}\text { Numerical solution - 4 } \\
\text { terms ADM }\end{array}$ & $\begin{array}{c}\text { Absolute Error } \\
(\%)\end{array}$ \\
\hline 0.001 & 2 & 2 & 2 & 0 & 0 \\
\hline 0.01 & 2.002999 & 2.002999 & 2.003 & 0.00000145 & 0.00007244 \\
\hline 0.1 & 2.0299 & 2.029854 & 2.0298 & -0.00005405 & 0.00266277 \\
\hline 0.5 & 2.2895 & 2.284589 & 2.2842 & -0.00038930 & 0.01704330 \\
\hline 1 & 3.1875 & 3.049955 & 3.049 & -0.00095492 & 0.03131915 \\
\hline
\end{tabular}

Table 4. Comparison of the numerical results with VIM solution for Van der Pol equation at $\varepsilon=0.1$ for different values of $t$.

\begin{tabular}{|c|c|c|c|c|}
\hline$t$ & $\begin{array}{c}2 \text { iterations of } \\
\text { VIM }\end{array}$ & $\begin{array}{c}\text { Numerical } \\
\text { (Runge-Kutta) }\end{array}$ & $\begin{array}{c}\text { Numerical solution - 2 } \\
\text { iterations of VIM }\end{array}$ & $\begin{array}{c}\text { Absolute Error } \\
(\%)\end{array}$ \\
\hline 0.001 & 2.002999 & 2 & 0.00000145 & 0.00007244 \\
\hline 0.01 & 2.029854 & 2.003 & -0.00005390 & 0.00265534 \\
\hline 0.1 & 2.284378 & 2.0298 & -0.00017750 & 0.00777077 \\
\hline 0.5 & 2.985938 & 2.2842 & 0.06306250 & 2.06830108 \\
\hline 1 & 2.225 & 3.049 & 0.93700000 & 29.63314358 \\
\hline
\end{tabular}

Table 5. Comparison of the numerical results with ADM and VIM solutions of Van der Pol equation at $\varepsilon=10$ for different values of $t$.

\begin{tabular}{|c|c|c|c|c|c|}
\hline$t$ & $\begin{array}{c}4 \text { terms } \\
\text { of ADM }\end{array}$ & $\begin{array}{c}2 \\
\text { iterations } \\
\text { of VIM }\end{array}$ & $\begin{array}{c}\text { Numerical } \\
\text { (Runge-Kutta) }\end{array}$ & $\begin{array}{c}\text { Difference between } \\
\text { Numerical solution } \\
\text { and ADM }\end{array}$ & $\begin{array}{c}\text { Difference between } \\
\text { Numerical solution } \\
\text { and VIM }\end{array}$ \\
\hline 0 & 2 & 2 & 2 & 0 & 0 \\
\hline 0.001 & 2.002954 & 2.002954 & 2.003 & 0.00004560 & 0.00229955 \\
\hline 0.01 & 2.02578 & 2.025339 & 2.0257 & -0.00008025 & 0.01780742 \\
\hline 0.1 & 1.699198 & 1.77725 & 2.0863 & 0.38710178 & 14.81330585 \\
\hline
\end{tabular}


Dynamics of the system under consideration are governed by the following set of partial differential equations:

$$
\begin{aligned}
& u_{t t}+a_{1}^{4} u_{x x x x}=k(v-u)-\beta_{1} u_{t}, \quad 0<x<1, t>0, \\
& v_{t t}+a_{2}^{4} v_{x x x x}=k(u-v)-\beta_{2} v_{t}, \quad 0<x<1, t>0,
\end{aligned}
$$

with initial conditions,

$$
\begin{aligned}
& u(x, 0)=p_{1}(x), \quad v(x, 0)=q_{1}(x) \quad 0 \leq x \leq 1 \\
& u_{t}(x, 0)=p_{2}(x), \quad v_{t}(x, 0)=q_{2}(x)
\end{aligned}
$$

and boundary conditions,

$$
\begin{array}{ll}
u(0, t)=u_{x x}(0, t)=0, & v(0, t)=v_{x x}(0, t)=0, \quad t>0 \\
u(1, t)=u_{x x}(1, t)=0, & v(1, t)=v_{x x}(1, t)=0
\end{array}
$$

where $t$ and $x$ represent the time and space variables, respectively, and $u=u(x, t)$ and $v=$ $v(x, t)$ are the vertical displacements of the beams measured from the horizontal equilibrium positions. The system parameters, $a_{i} \geq 0, i=1,2$, are described in terms of flexural rigidity coefficient, $E_{i} I_{i}$, and mass density, $m_{i}$, as $a_{i}^{4}=E_{i} I_{i} / m_{i}$, where $E_{i}$ denotes Young modulus of elasticity and $I_{i}$ denotes the cross-sectional area. Uniform beam properties are assumed; that is, $m_{i}, E_{i}$ and $I_{i}$ are constants. The terms $\pm k(u-v)$ represent the coupling between the two beams and $k$ denotes the elastic coupling constant. The terms $-\beta_{1} u_{t}$ and $-\beta_{2} v_{t}$ represent the velocity feedback controllers.

Having considered the procedures in Section 2 for ADM, one can rewrite (51) in the following operator form:

$$
\left\{\begin{array}{l}
L_{t}(u)+a_{1}^{4} L_{x}(u)+R_{1}(u, v)=0, \\
L_{t}(v)+a_{2}^{4} L_{x}(v)+R_{2}(u, v)=0 .
\end{array}\right.
$$

The linear term is decomposed to $L$ and $R$, where $L$ is the highest order derivative and $R$ is the reminder of the linear operator. $L_{t}$ and $L_{x}$ are considered second order and forth-partial differential operator in $t$ and $x$, respectively. $L_{t}^{-1}$ is twofold integration with respect to $t$ from 0 to $t$. Applying the $L_{t}^{-1}$ to system (54) and using initial conditions (52) yields

$$
\begin{aligned}
& u(x, t)=p_{1}(x)+t p_{2}(x)-a_{1}^{4} L_{t}^{-1} L_{x}(u)-L_{t}^{-1} R_{1}(u, v), \\
& v(x, t)=q_{1}(x)+t q_{2}(x)-a_{2}^{4} L_{t}^{-1} L_{x}(v)-L_{t}^{-1} R_{2}(u, v) .
\end{aligned}
$$

The first two terms of (55) are integration constants. According to ADM, $u(x, t)$ and $v(x, t)$ can be decomposed as follows:

$$
\begin{aligned}
& u(x, t)=\sum_{n=0}^{\infty} u_{n}(x, t), \\
& v(x, t)=\sum_{n=0}^{\infty} v_{n}(x, t) .
\end{aligned}
$$


Substituting (56) into (55) gives

$$
\begin{aligned}
& \sum_{n=0}^{\infty} u_{n}(x, t)=p_{1}(x)+t p_{2}(x)-a_{1}^{4} L_{t}^{-1} L_{x}\left(\sum_{n=0}^{\infty} u_{n}(x, t)\right)-L_{t}^{-1} R_{1}\left(\sum_{n=0}^{\infty} u_{n}(x, t), \sum_{n=0}^{\infty} v_{n}(x, t)\right), \\
& \sum_{n=0}^{\infty} v_{n}(x, t)=q_{1}(x)+t q_{2}(x)-a_{1}^{4} L_{t}^{-1} L_{x}\left(\sum_{n=0}^{\infty} v_{n}(x, t)\right)-L_{t}^{-1} R_{2}\left(\sum_{n=0}^{\infty} u_{n}(x, t), \sum_{n=0}^{\infty} v_{n}(x, t)\right),
\end{aligned}
$$

The system in (57) is transformed to a set of recursive relations given by

$$
\begin{aligned}
& u_{0}(x, t)=p_{1}(x)+t p_{2}(x), \\
& u_{n+1}(x, t)=-a_{1}^{4} L_{t}^{-1} L_{x}\left(u_{n}\right)-L_{t}^{-1} R_{1}\left(u_{n}, v_{n}\right), \quad n \geq 0,
\end{aligned}
$$

and similarly

$$
\begin{aligned}
& v_{0}(x, t)=q_{1}(x)+t q_{2}(x), \\
& v_{n+1}(x, t)=-a_{2}^{4} L_{t}^{-1} L_{x}\left(v_{n}\right)-L_{t}^{-1} R_{1}\left(u_{n}, v_{n}\right), \quad n \geq 0 .
\end{aligned}
$$

Here, $u_{0}$ and $v_{0}$ can be obtained using (52). The terms $u_{n+1}$ and $v_{n+1}$ are calculated using preceding terms. Consequently, the summation of $u_{n}$ and $v_{n}$ terms is the desired solution which converges rapidly. In real world, we can calculate $k$ terms of the summation, so the analytical approximate solution is

$$
\varphi_{k}=\sum_{n=0}^{k-1} u_{n}(x, t), \quad \psi_{k}=\sum_{n=0}^{k-1} v_{n}(x, t) .
$$




\section{Examples}

Example 1. The system we are dealing with is a parallel system of Euler-Bernoulli beams with distributed springs and dampers. Without loss of generality, let $k=\beta_{1}=\beta_{2}=1$ and also $a_{1}=a_{2}=1$, then (51) becomes

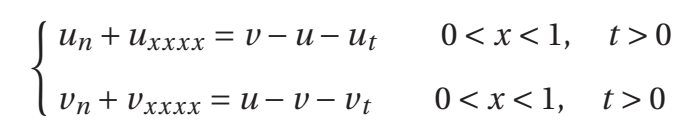

and initial conditions are

$$
\begin{array}{ll}
u(x, 0)=p_{1}(x)=\sin (\pi x), & v(x, 0)=q_{1}(x)=-\sin (\pi x), \\
u_{t}(x, 0)=p_{2}(x)=0, & v_{t}(x, 0)=q_{2}(x)=0,
\end{array}
$$

and with prescribed boundary conditions

$$
\begin{array}{ll}
u(0, t)=u_{x x}(0, t)=0, & v(0, t)=v_{x x}(0, t)=0 \\
u(1, t)=u_{x x}(1, t)=0, & v(1, t)=v_{x x}(1, t)=0 .
\end{array}
$$

After decomposition $u(x, t)$ and $v(x, t)$, according to (57), the system (61) can be rewritten as

$$
\begin{aligned}
& \sum_{n=0}^{\infty} u_{n}(x, t)=\sin (\pi x)+\iint\left[-\left(\sum_{n=0}^{\infty} u_{n}\right)_{x x x x}+\sum_{n=0}^{\infty} v_{n}-\sum_{n=0}^{\infty} u_{n}-\left(\sum_{n=0}^{\infty} u_{n}\right)_{t}\right] d t d t \\
& \sum_{n=0}^{\infty} v_{n}(x, t)=-\sin (\pi x)+\iint\left[-\left(\sum_{n=0}^{\infty} v_{n}\right)_{x x x x}+\sum_{n=0}^{\infty} u_{n}-\sum_{n=0}^{\infty} v_{n}-\left(\sum_{n=0}^{\infty} v_{n}\right)_{t}\right] d t d t
\end{aligned}
$$

Each of equations in (64) can be rewritten in a set of the following recrusive relations:

$$
\begin{aligned}
& u_{0}(x, t)=\sin (\pi x) \\
& u_{n+1}(x, t)=\iint\left[-\left(u_{n}\right)_{x x x x}+v_{n}-u_{n}-\left(u_{n}\right)_{t}\right] d t d t, \quad n \geq 0 .
\end{aligned}
$$

Similarly,

$$
\begin{aligned}
& v_{0}(x, t)=-\sin (\pi x), \\
& v_{n+1}(x, t)=\iint\left[-\left(v_{n}\right)_{x x x x}+u_{n}-v_{n}-\left(v_{n}\right)_{t}\right] d t d t, \quad n \geq 0 .
\end{aligned}
$$

Now, having considered (60), one can find the following analytical approximate solution for $u$ and $v$, respectively: 


$$
\begin{aligned}
& \varphi_{3}=\sum_{n=0}^{2} u_{n}(t)=\left[1-\frac{1}{2}\left(\pi^{4}+2\right) t^{2}+\frac{1}{6}\left(\pi^{4}+2\right) t^{3}+\frac{1}{24}\left(\pi^{8}+4 \pi^{4}+4\right) t^{4}\right] \sin (\pi x), \\
& \psi_{3}=\sum_{n=0}^{2} v_{n}(t)=-\left[1-\frac{1}{2}\left(\pi^{4}+2\right) t^{2}+\frac{1}{6}\left(\pi^{4}+2\right) t^{3}+\frac{1}{24}\left(\pi^{8}+4 \pi^{4}+4\right) t^{4}\right] \sin (\pi x) .
\end{aligned}
$$

Our attempt is now to find the closed form solution to system (61). To do this, we use Aftertreatment (AT) technique by Jiao (2002) that leads to a closed form solution. This technique uses Laplace transform and Padé approximation (Adomian, G., Kluwer Academic, 1994), which approximates a function by ratio of two polynomials. Due to AT technique, Laplace transform is applied to the coefficients of $\sin (\pi x)$ in (67) yields

$$
\ell\left(\varphi_{3}(x, t)\right)=\left\{\frac{1}{s^{5}}\left[\left(\pi^{8}+4 \pi^{4}+\pi\right)+\left(\pi^{4}+2\right) s-\left(\pi^{4}+2\right) s^{2}+s^{4}\right]\right\} \sin \pi x .
$$

For the sake of simplicity, let $s=1 / \xi$; then (68) becomes

$$
\bar{\ell}\left(\varphi_{3}(x, t)\right)=\left\{\left[\xi-\left(\pi^{4}+2\right) \xi^{3}+\left(\pi^{4}+2\right) \xi^{4}+\left(\pi^{8}+4 \pi^{4}+4\right)\right] \xi^{5}\right\} \sin \pi x .
$$

Equation (69) is now approximated by Padé approximation $\left[\frac{2}{2}\right]$ yields

$$
\left[\frac{2}{2}\right]_{\xi}=\frac{\xi(1+\xi)}{1+\xi+\left(2+\pi^{4}\right) \xi^{2}} \text {. }
$$

Let $\xi=1 / s$, then (70) becomes

$$
\left[\frac{2}{2}\right]_{s}=\frac{s+s^{2}}{\left(2+\pi^{2}\right)+s+s^{2}} .
$$

Finally, applying the inverse Laplace transform to (71) results to the following analytical approximate solution to the system (61) for $u(x, t)$ :

$$
u(x, t)=e^{\left(-\frac{t}{2}\right)}\left[\cosh \left(\frac{1}{2} \sqrt{-7-4 \pi^{4}}\right) t+\frac{\sinh \left(\frac{1}{2} \sqrt{-7-4 \pi^{4}}\right) t}{\left(\sqrt{-7-4 \pi^{4}}\right)}\right] \sin (\pi x) .
$$

Similarly for $v(x, t)$,

$$
v(x, t)=-e^{\left(-\frac{t}{2}\right)}\left[\cosh \left(\frac{1}{2} \sqrt{-7-4 \pi^{4}}\right) t+\frac{\sinh \left(\frac{1}{2} \sqrt{-7-4 \pi^{4}}\right) t}{\left(\sqrt{-7-4 \pi^{4}}\right)}\right] \sin (\pi x) .
$$

Due to the bounded ness of the complex hyperbolic trigonometric functions as well as sine function, one can see from (72) and (73) that as $t \rightarrow \infty$, the solutions $u, v \rightarrow 0$, which implies that the system (61) is stable, as it was proved by Najafi (Journal of Vibration and Control. Vol. $3,1997)$.

Example 2. The governing equation for coupled wave equations in $\Omega \subset \mathfrak{R}^{2}$ and boundary $\partial \Omega$ is as follows:

$$
u_{n}=c_{1}^{2} \Delta u+k(v-u)-\beta_{1} u_{t}
$$




$$
v_{n}=c_{2}^{2} \Delta v+k(u-v)-\beta_{2} v_{t}, \quad \text { in } \Omega \times(0, \infty),
$$

with initial conditions,

$$
\begin{aligned}
& u(0)=v(0)=\sin (\pi x) \sin (\pi y), \quad \text { in } \Omega \\
& u_{t}(0)=v_{t}(0)=0,
\end{aligned}
$$

and boundary conditions,

$$
u=v=0, \quad \text { on } \partial \Omega \times(0, \infty) .
$$

Having considered the procedures in Section 2 for ADM and following the steps in Section 6 , one can rewrite (74) in the following operator form:

$$
\begin{aligned}
& L_{t} u=c_{1}^{2} L u+R_{1}(u, v), \\
& L_{t} v=c_{2}^{2} L v+R_{1}(u, v),
\end{aligned}
$$

where $L_{t}=\frac{\partial^{2}(\cdot)}{t^{2}}, L=\frac{\partial^{2}(\cdot)}{x^{2}}+\frac{\partial^{2}(\cdot)}{y^{2}}$, and $R$ is the reminder of the linear Operator. Applying $L_{t}^{-1}$, which is twofold integration with respect to $t$ from 0 to $t$, to system (76) and using initial conditions (75) yields

$$
\begin{aligned}
& u=\sin (\pi x) \sin (\pi y)+c_{1}^{2} L_{t}^{-1} L(u)-L_{t}^{-1} R_{1}(u, v), \\
& \nu=\sin (\pi x) \sin (\pi y)+c_{2}^{2} L_{t}^{-1} L(\nu)-L_{t}^{-1} R_{2}(u, v) .
\end{aligned}
$$

According to ADM, $u$ and $v$ can be decomposed as follows:

$$
u=\sum_{0}^{\infty} u_{n}, \quad v=\sum_{0}^{\infty} v_{n} .
$$

Substituting (78) into (77) gives

$$
\begin{aligned}
u= & \sum_{n=0}^{\infty} u=\sin (\pi x) \sin (\pi y)+\iint\left[c_{1}^{2}\left[\left(\sum_{n=0}^{\infty} u_{n}\right)_{x x}+\left(\sum_{n=0}^{\infty} u_{n}\right)_{y y}\right]\right. \\
& \left.+k\left[\sum_{n=0}^{\infty} v_{n}-\sum_{n=0}^{\infty} u_{n}\right]-\beta_{1}\left(\sum_{n=0}^{\infty} u_{n}\right)_{t}\right] d t d t, \\
v= & \sum_{n=0}^{\infty} v=\sin (\pi x) \sin (\pi y)+\iint\left[c_{2}^{2}\left[\left(\sum_{n=0}^{\infty} v_{n}\right)_{x x}+\left(\sum_{n=0}^{\infty} v_{n}\right)_{y y}\right]\right. \\
& \left.+k\left[\sum_{n=0}^{\infty} u_{n}-\sum_{n=0}^{\infty} v_{n}\right]-\beta_{2}\left(\sum_{n=0}^{\infty} v_{n}\right)_{t}\right] d t d t .
\end{aligned}
$$

Each of equations in (79) can be rewritten in a set of the following recursive relations:

$$
\begin{aligned}
& u_{0}=\sin (\pi x) \sin (\pi y), \\
& u_{n+1}=\iint\left[c_{1}^{2}\left(\left(u_{n}\right)_{x x}+\left(u_{n}\right)_{y y}\right)+k\left[v_{n}-u_{n}\right]-\beta_{1}\left(u_{n}\right)_{t}\right] d t d t, n \geq 0 .
\end{aligned}
$$


Similarly,

$$
\begin{aligned}
& v_{0}=\sin (\pi x) \sin (\pi y), \\
& v_{n+1}=\iint\left[c_{2}^{2}\left(\left(v_{n}\right)_{x x}+\left(v_{n}\right)_{y y}\right)+k\left[u_{n}-v_{n}\right]-\beta_{2}\left(v_{n}\right)_{t}\right] d t d t, n \geq 0 .
\end{aligned}
$$

Here, $u_{0}$ and $v_{0}$ can be obtained using (75). The terms $u_{n+1}$ and $v_{n+1}$ are calculated using preceding terms. Consequently, the summation of $u_{n}$ and $v_{n}$ terms is the desired solution which converges rapidly. We can calculate $k$ terms of the summation to find approximate analytical solution as:

$$
\varphi_{k}=\sum_{n=0}^{k-1} u_{n}, \quad \psi_{k}=\sum_{n=0}^{\infty} v_{n}
$$

For the purpose of the calculation, let, without loss of generality, $c_{1}=c_{2}=1$ and $k=\beta_{1}=\beta_{2}=$ 1 , then the following calculations are the first three terms of (80):

$$
\begin{aligned}
& u_{1}=-\sin (\pi x) \sin (\pi y) \pi^{2} t^{2}, \\
& u_{2}=\sin (\pi x) \sin (\pi y)\left[\frac{1}{6} \pi^{4} t^{4}+\frac{1}{3} \pi^{2} t^{3}\right], \\
& u_{3}=\sin (\pi x) \sin (\pi y)\left[-\frac{1}{90} \pi^{6} t^{6}-\frac{1}{15} \pi^{4} t^{5}-\frac{1}{12} \pi^{2} t^{4}\right] .
\end{aligned}
$$

From (82), one can write the following analytical approximate solution for (74):

$$
\begin{aligned}
& \varphi_{4}=\sin (\pi x) \sin (\pi y)\left[1-\pi^{2} t^{2}+\frac{1}{3} \pi^{2} t^{3}+\frac{1}{15} \pi^{4} t^{4}-\frac{1}{6} \pi^{2} t^{4}-\frac{1}{15} \pi^{4} t^{5}-\frac{1}{90} \pi^{6} t^{6}\right], \\
& \psi_{4}=\sin (\pi x) \sin (\pi y)\left[1-\pi^{2} t^{2}+\frac{1}{3} \pi^{2} t^{3}+\frac{1}{15} \pi^{4} t^{4}-\frac{1}{6} \pi^{2} t^{4}-\frac{1}{15} \pi^{4} t^{5}-\frac{1}{90} \pi^{6} t^{6}\right] .
\end{aligned}
$$

Dur to AT technique, see Example 1, Laplace transform is applied to the coefficiets of $\sin (\pi x) \sin (\pi y)$ in (84) yields

$$
\begin{aligned}
\ell\left(\varphi_{4}\right)= & \left\{\frac { 1 } { s ^ { 7 } } \left[-\left(4 \pi^{2}+8 \pi^{4}+8 \pi^{6}\right)-\left(8 \pi^{4}+4 \pi^{2}\right) s+4 s^{2}+2 \pi^{2} s^{3}\right.\right. \\
& \left.\left.-2 \pi^{2} s^{4}+s^{6}\right]\right\} \sin (\pi x) \sin (\pi y) .
\end{aligned}
$$

For the sake of simplicity, let $s=1 / \xi$; then (85) becomes

$$
\begin{aligned}
\bar{\ell}\left(\varphi_{4}\right)= & {\left[-\left(4 \pi^{2}+8 \pi^{4}+8 \pi^{6}\right) \xi^{7}-\left(8 \pi^{4}+4 \pi^{2}\right) \xi^{6}+4 \xi^{5}+2 \pi^{2} \xi^{4}\right.} \\
& \left.\left.-2 \pi^{2} \xi^{3}+\xi\right]\right\} \sin (\pi x) \sin (\pi y) .
\end{aligned}
$$

Equation (86) is now approximated by Padé approximation $\left[\frac{2}{2}\right]$ yields

$$
\left[\frac{2}{2}\right]_{\xi}=\frac{\xi(1+\xi)}{1+\xi+2 \pi^{2} \xi^{2}}
$$


Let $\xi=1 / s$, then (87) becomes

$$
\left[\frac{2}{2}\right]_{s}=\frac{s+1}{s^{2}+s+2 \pi^{2}}
$$

Finally, applying the inverse Laplace transform to (88) results to the following analytical approximate solution in the closed form for (74) in $\mathfrak{R}^{2}$, respectively:

$$
u=e^{\left(-\frac{t}{2}\right)}\left[\cos \left(\frac{1}{2} \sqrt{-1+8 \pi^{2}}\right) t+\frac{\sin \left(\frac{1}{2} \sqrt{-1+8 \pi^{2}}\right) t}{(\sqrt{-1+8 \pi})}\right] \sin (\pi x) \sin (\pi y) .
$$

Similarly for $v$,

$$
v=e^{\left(-\frac{t}{2}\right)}\left[\cos \left(\frac{1}{2} \sqrt{-1+8 \pi^{2}}\right) t+\frac{\sin \left(\frac{1}{2} \sqrt{-1+8 \pi^{2}}\right) t}{(\sqrt{-1+8 \pi})}\right] \sin (\pi x) \sin (\pi y) .
$$

One can see from above solutions that $u, v \rightarrow 0$, as $t \rightarrow \infty$, which implies that the system in (74) is a stabilized system. This phenomenon was expected to happen, since (74) is exposed to the velocity feedback controllers, see Najafi (IEEE Transaction on Automatic Control. Vol. 42, 1997).

\section{Conclusion}

In this paper, the standard Adomian Decomposition Mehod (ADM) is used, which is the focal point of this work, to solve nonlinear oscillation equations such as Duffing and Van der Pol equations analytically. In addition, we found the solution of the system of coupled wave equations in a closed form and by using the initial conditions only. In comparison with perturbation or linearization methods, this method gives the analytical solution in series form which converges rapidly. Unlike other common methods for solving any physical problem, linear or nonlinear, ADM solves many types of problems without requiring linearization, discretization, perturbation, or unjustified assumptions that may slightly change the physics of the problem. For a large number of problems, the decomposition method has shown reliable results in providing analytical approximation that converges rapidly.

\section{References}

[1] K. Abbaoui and Y. Cherruault, Convergence of Adomian's method applied to differentical equations, Comput. Math. Appl. 102(1999), 77-86.

[2] G. Adomian, Solving Frontier Problems of Physics: The Decomposition Method, Kluwer Academic, 1994.

[3] G. Adomian, Solution of physical problems by decomposition, Computers Math. Application 27(1994), 145-154.

[4] G. Adomian, A review of the decomposition method in applied mathematics, J. Math. Anal. Appl. 135(1988), 501-544.

[5] J. H. He, A new approach to nonlinear partial differential equations, Communications inNonlinear Science and Numerical Simulation 2(1997), 230-235.

[6] J. H. He, Approximate analytical solution for seepage flow with fractional derivatives in porous media, Computer Methods in Applied Mechanics and Engineering 167(1998), 57-68. 
[7] J. H. He, Variational iteration method - a kind of non-linear analytical technique: some examples, International Journal of Non-Linear Mechanics 34(1999), 699-708.

[8] Y. C. Jiao, et al., An aftertreatment technique for improving the accruacy of Adomian's decomposition method, Computers Math. Applic. 43(2002), 783-798.

[9] M. Najafi, G. R. Sarhangi and H. Wang, Stabilizability of coupled wave equation in parallel under various boundary conditions, IEEE Transaction on Automatic Control 42(1997), 1308-1312.

[10] M. Najafi, G. R. Sarhangi and H. Oloomi, Distributed and boundary control for a parallel system of Euler-Bernoulli beams, Journal of Vibration and Control 3(1997), 183-199.

[11] M. Najafi, Study of Exponentical stability of coupled wave system via distributed stabilizer, International Journal of Mathematics and Mathematical Science (2001), 479-491.

[12] S. Pamuk, Solution of the porous media equation by Adomian's decomposition method, Physics Letters A. 344(2005), 184-188.

[13] M. F. White, Viscous Fluid Flow, $2^{\text {nd }}$ Ed., McGraw-Hill, Inc., 1991.

Department of Mathematics, Kent State University, 3300 Lake Road West, Ashtabula, OH 44004, U.S.A. Acoustics Research Center, Institute of Applied Physics, Tehran, Iran.

E-mail: mnajafi@kent.edu

Acoustics Research Center, Institute of Applied Physics, Tehran, Iran.

Department of Mechanical Engineering, University of Science and Technology, Tehran, Iran.

Acoustics Research Center, Institute of Applied Physics, Tehran, Iran.

Department of Physics, Amirkabir University, Tehran, Iran. 